\title{
Short-Term Follow-Up of Self-Isolated COVID-19 Patients with Smell and Taste Dysfunction in Greece: Two Phenotypes of Recovery
}

\author{
Iordanis Konstantinidis $^{a} \quad$ Alexander Delides $^{b} \quad$ Evangelia Tsakiropoulou $^{a}$ \\ Pavlos Maragoudakis $^{b}$ Spyridon Sapounas ${ }^{c}$ Sotirios Tsiodras ${ }^{d}$ \\ a2nd Academic ORL Department, Papageorgiou Hospital, Aristotle University, Thessaloniki, \\ Greece; ${ }^{b}$ 2nd Academic ORL Department, Attikon University Hospital, School of Medicine, \\ National and Kapodistrian University of Athens, Athens, Greece; ${ }^{C}$ National Public Health \\ Organization, Athens, Greece; ${ }^{d}$ 4th Department of Internal Medicine, Attikon University \\ Hospital, School of Medicine, National and Kapodistrian University of Athens, Athens, \\ Greece
}

\author{
Keywords \\ COVID-19 - Olfactory dysfunction · Gustatory dysfunction · Smell · Taste
}

\begin{abstract}
Introduction: The course of anosmia and ageusia in COVID-19 patients is not yet clearly known. We present short-term follow-up data concerning mild to moderate disease in homequarantined COVID-19 patients in Greece. Methods: We provided a symptom questionnaire and instructions for a self-administered home smell-and-taste test to 79 positive COVID-19 patients from 2 tertiary hospitals in Greece. The patients recorded their subjective symptoms before and during infection as well as 4 weeks after the diagnosis. The patients also underwent the home test during infection and 4 weeks later. Results: Twenty-nine patients (36.7\%) reported a loss of smell, and 21 (27.8\%) reported a loss of taste, with equal prevalences between genders. We observed 2 types of recovery, i.e., a rapid, almost complete recovery, and a second slower and partial recovery. The type of recovery was not age related. A rapid recovery was observed in two thirds of the patients, with their olfactory ratings presenting a trend towards significance in correlation with nasal obstruction. A slow recovery in olfaction was correlated with low intensity ratings in odors with a trigeminal compound. The loss of taste was more pronounced in sweet and salty intensity ratings. Conclusion: Chemosensory deficits associated with COVID-19 infection were quite frequent among the Greek patients with mild or moderate disease who, in most cases, returned to normal within 4 weeks. However, 1 in 3 patients presented with persistent olfactory and gustatory dysfunction in the short term.


Patients

\section{Introduction}

Olfactory and gustatory dysfunctions have been recognized by the World Health Organization (WHO) as symptoms of COVID-19 infection. The estimated prevalence ranges from 18 to $60 \%$ [1-3]. However, insufficient data exists regarding the course and prognosis of this dysfunction.

According to the disease control policy of Greece, patients with a laboratory-confirmed diagnosis and a mild or moderate COVID-19 infection are advised to engage in self-isolation under the close supervision of medical professionals. Chemosensory dysfunction appears to be a common symptom in the early stages of COVID-19 infection. Thus, patients with an early diagnosis can be found and followed up for smell and taste deficits more easily among those with mild-to-moderate disease rather than among hospitalized patients.

Psycho-physical tests are the gold standard in everyday clinical practice for the evaluation of olfactory and gustatory functions, as they are easily performed, standardized, and validated in many languages, with a high test-retest reliability [4-6]. However, during these tests, patients are blinded to the presented items. This makes home testing difficult, especially if we ask patients to self-administer odors and tastants.

A more straight-forward test appears to be the assessment of smell and taste intensities of common household items, since this avoids the limitations of a self-administered identification smell-and-taste test. Previous publications have used odor and tastant intensities as a measure of olfactory and gustatory function, respectively $[7,8]$. At least in the case of olfaction, this was found to be related to odor detection thresholds concerning the odor in question [9]. In a recent study by Iravani et al. [10], in 2,440 Swedish individuals, measures of odor intensity closely tracked the estimated COVID-19 levels at a population level. However, as the testretest reliability of odor intensity measures presents large variations $[9,10]$, this may indicate that odor intensity function might not work as a reliable measure at the individual level.

Our study aims to present a first estimation of the prognosis of home-quarantined patients with a positive nasal or pharyngeal swab for COVID-19 (real-time polymerase chain reaction; RT-PCR) and a mild or moderate disease severity by analyzing follow-up data 4 weeks after the diagnosis. This study also combines subjective ratings of patients' symptoms based on a self-administered home test of olfactory and gustatory functions to provide a more objective chemosensory assessment of home-quarantined COVID-19 patients.

\section{Materials and Methods}

\section{Patients}

The National testing policy at the beginning of the pandemic in Greece focused on tracking and tracing of: (1) new clinically positive cases requiring a hospital stay, and (2) clinically positive cases from the country's entry points to suppress local outbreaks. A special task healthcare team of the General Secretariat for Civil Protection (GCSP) and the National Public Health Organization (NPHO) obtained nasal/pharyngeal swabs for RT-PCR from all of the close contacts of the above cases. These data were stored in a national database developed by the NPHO.

Over a period of 2 months (from March 22 to May 15, 2020) we retrieved from this database all newly diagnosed patients with mild-to-moderate disease according to the national guidelines for primary care against COVID-19 in Greece (www.eody.gov.gr), who had instructed to stay home quarantined and followed by the 2 contributing tertiary hospitals in Greece.

As regular daily communication of self-quarantine patients with a healthcare professional was advised throughout the monitoring period, both hospitals followed: (1) self-referred patients with a positive test from elsewhere and examined in their emergencies, (2) positive close contacts of hospitalized patients, and (3) positive repatriated passengers from certain countries with presumed widespread SARSCoV-2 infection. 
Konstantinidis et al.: Short-Term Follow-Up of Chemosensory Dysfunction in COVID-19 Patients

A hard copy of an anonymous smell-and-taste questionnaire along with instructions for a home test of olfactory and gustatory function was administered and explained to 300 adult patients at their homes by the health care personnel of the NPHO and the GCSP. The patients had to fill in the questionnaire at the baseline visit of the NPHO and GCSP team and then, if the RT-PCR test was positive, they had to refill it 4 weeks later. The questionnaires were collected by the NPHO and GCSP personnel the end of the quarantine period or mailed directly to the hospitals.

Exclusion criteria were: age $<18$ years, previous nasal surgery, radiotherapy in the head and neck area, a history of head trauma, allergic rhinitis, chronic rhinosinusitis, psychiatric or neurological disorders, and a history of previous smell-and-taste disorders.

Patient demographics, associated symptoms, and comorbidities were recorded. The patients had to report symptoms from the following list: fever, cough, shortness of breath or dyspnea, fatigue, muscle aches, runny nose, blocked nose, sore throat, loss of smell, and loss of taste.

\section{Subjective Ratings of Olfaction and Taste}

The patients had to rate their olfactory and tasting abilities by means of a visual analog scale (VAS) at the time of diagnosis and 4 weeks later. They were also asked to evaluate their capacity to smell and taste before infection, as they could recall it. Ratings were recorded on a horizontally presented 100-point VAS, where 0 represents no smell or taste and 100 normal sensation. In addition, patients rated their nasal obstruction and rhinorrhea on a similar VAS, where 0 represented a completely blocked nose and an excessively runny nose and 100 indicated normal nasal breathing and a nonrunny nose, respectively. The patients also reported the presence of qualitative olfactory and gustatory dysfunction. Four weeks after the diagnosis, all of the patients stated whether they had experienced complete recovery or partial/no recovery in terms of chemosensory deficits.

Intensity Ratings: Olfactory and Gustatory Home Test

All of the participants were instructed to rate the intensity of their olfactory ability in response to 5 common household odors (i.e., lemon juice, oregano, instant coffee, toothpaste, and mint gum). The rating range was from 0 (no smell) to 100 (smell as usual). The first 3 odors were selected to contain odors with little or no trigeminal sensation (unimodal odors), whereas the last 2 odors could evoke mixed sensations of olfactory and trigeminal nerves (bimodal odors). The patients could smell each odor as often as they liked without time limitations.

For intensity ratings of the gustatory function, sugar, salt, lemon juice, and instant coffee (decaffeinated) were used as representatives of the following 4 basic taste qualities: sweet, salty, sour, and bitter.

The patients were asked to put in their mouth half a teaspoon of each tastant, report the quality of taste perception, and then rinse their mouth with tap water. The patients were instructed to taste the bitter tastant last in the order [11]. The intensity score ranged from 0 (no taste) to 100 (normal perception). A mean of the reported values for each of the 5 odors and 4 tastes was calculated as the smell and taste overall intensity score, respectively.

\section{Statistical Analysis}

All demographics are presented within the text as means \pm SD and ranges of values. The $\alpha$-level of significance was set to 0.05. Paired two-tailed Student $t$ tests were used to assess VAS and odor intensity ratings before and during infection and 4 weeks later. All correlation tests were performed using Spearman correlation analysis.

\section{Results}

In total, 145 questionnaires out of 300 were returned (48.3\%). Thirty-seven were excluded due to a previous history of sinonasal disease or nasal surgery, and 29 were excluded due to incomplete data. Finally, 79 patients (39 males and 40 females) were enrolled into the present study.

Thirty of the 79 included patients reported a chemosensory deficit. Specifically, 21 patients complained of a loss of smell and taste, 8 patients reported only a loss of smell, and 


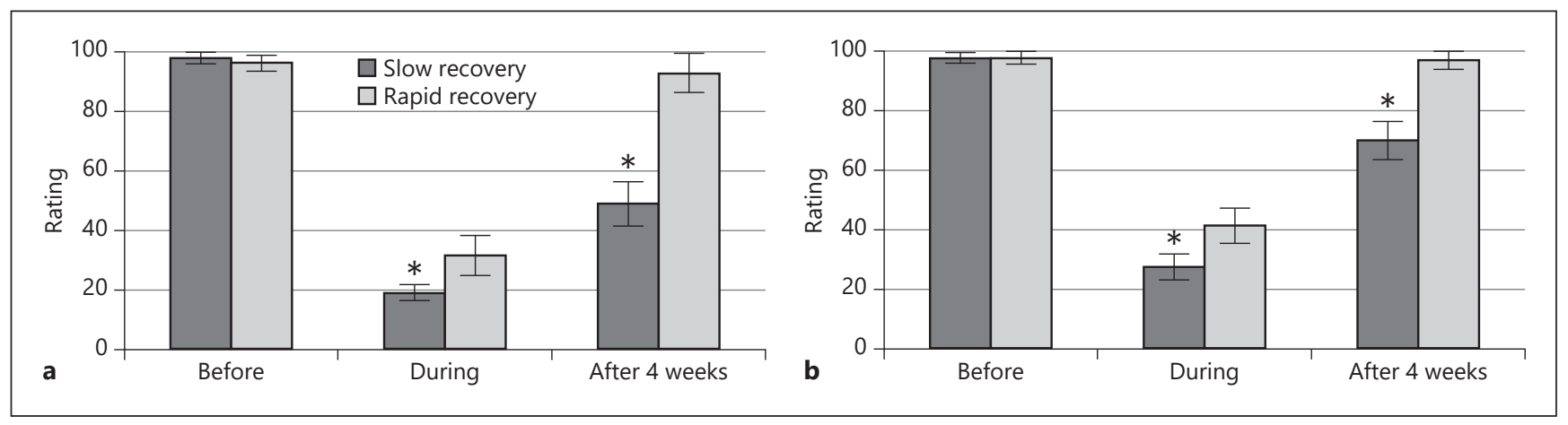

Fig. 1. Comparison of VAS olfactory function ratings (a) and VAS gustatory function ratings (b) between fast and slow recovery subgroups of patients before, during infection and 4 weeks later. The asterisk indicates statistical significance $(p<0.05)$.

Table 1. Patients' demographics and total group ratings

\begin{tabular}{|c|c|c|c|}
\hline \multicolumn{2}{|l|}{ Variable } & \multicolumn{2}{|l|}{ Value } \\
\hline \multicolumn{2}{|c|}{ Patients with a chemosensory loss } & \multicolumn{2}{|c|}{30 (36.7) } \\
\hline \multicolumn{2}{|c|}{$\mathrm{M} / \mathrm{F}$ ratio } & \multicolumn{2}{|c|}{ 16/14 (53.3/46.7) } \\
\hline \multicolumn{2}{|c|}{ Age, years } & \multicolumn{2}{|c|}{$30.7 \pm 5.3$} \\
\hline \multicolumn{4}{|c|}{ Type of chemosensory loss ${ }^{a}$} \\
\hline Only smell & & \multicolumn{2}{|c|}{$8(26.6)$} \\
\hline Smell and taste & & \multicolumn{2}{|c|}{$21(70.0)$} \\
\hline Only taste & & \multicolumn{2}{|c|}{$1(3.3)$} \\
\hline Patients with nasal & & \multicolumn{2}{|c|}{$5(16.6)$} \\
\hline Patients with rhino & & \multicolumn{2}{|c|}{$3(10.0)$} \\
\hline Patients with como & & \multicolumn{2}{|c|}{$4(13.3)$} \\
\hline Patients with mild $c$ & & \multicolumn{2}{|c|}{$25(83.3)$} \\
\hline \multicolumn{2}{|c|}{ Patients with moderate disease ${ }^{\mathrm{a}}$} & \multicolumn{2}{|c|}{$5(16.6)$} \\
\hline Ratings & Before & During & After \\
\hline VAS olfaction & $96.4 \pm 2.4$ & $27.5 \pm 5.1$ & $77.6 \pm 6.7$ \\
\hline VAS taste & $97.5 \pm 2.0$ & $36.7 \pm 5.3$ & $87.7 \pm 4.7$ \\
\hline Intensity olfaction & & $38.3 \pm 5.4$ & $71.3 \pm 6.4$ \\
\hline Intensity taste & & $43.8 \pm 5.3$ & $71.6 \pm 6.5$ \\
\hline
\end{tabular}

The total number of patients was 79. Values are presented as numbers (\%) or means \pm SD unless otherwise stated. ${ }^{\text {a Among the }} 30$ patients who reported a chemosensory loss.

1 patient reported only a loss of taste (loss of smell: 29 out of 79 patients, $36.7 \%$; loss of taste: 22 out of 79 patients, $27.8 \%$ ).

The gender distribution was 16 male and 14 female patients, with a mean age of $30.7 \pm$ 5.3 years. This cohort of patients rated their olfactory and gustatory abilities during infection as significantly lower than before (comparison of VAS means, all $p<0.001$ ). Four weeks after the diagnosis, they presented with significant improvement overall (comparison of VAS means, all $p<0.001$ ). However, the patients' ratings 4 weeks after the initial assessment did not return to the levels before infection, being significantly lower in olfaction ( $p=0.023)$ and taste $(p=0.037)$. The demographics and total study group statistics are presented in Table 1 . 
Konstantinidis et al.: Short-Term Follow-Up of Chemosensory Dysfunction in COVID-19 Patients

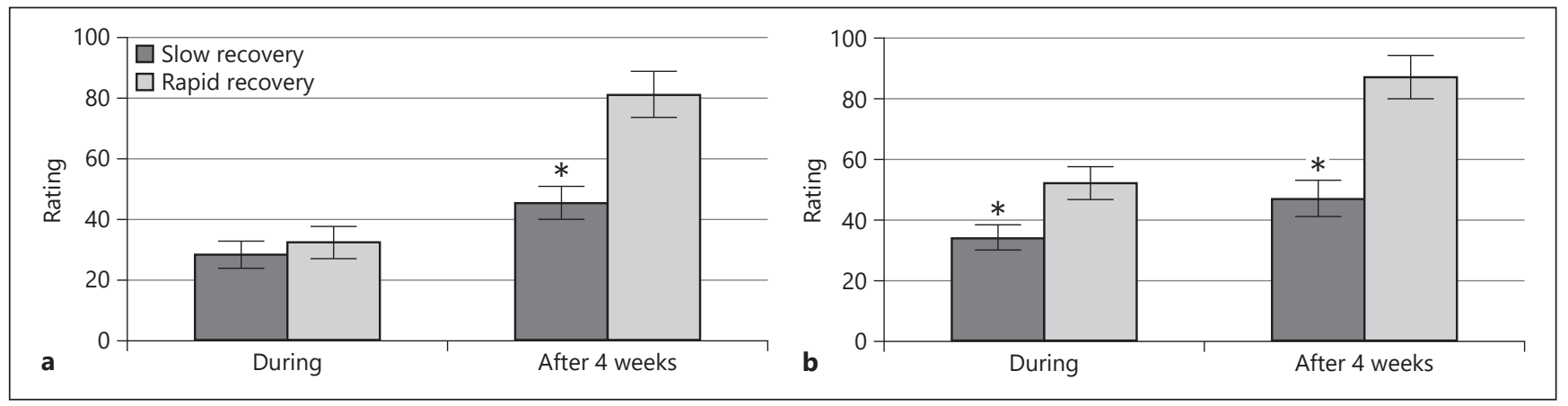

Fig. 2. Comparison of olfactory function intensity ratings between the rapid- and slow-recovery subgroups with unimodal odors (a) and with bimodal odors (b) before, during infection and 4 weeks later. The asterisk indicates statistical significance $(p<0.05)$.

A complete or almost complete recovery was reported by 19 patients (rapid-recovery subgroup, 63.3\%). However, 11 patients reported partial or no improvement (slow-recovery subgroup, $36.6 \%$ ), presenting a slow pattern of recovery.

A comparison of olfactory and gustatory VAS ratings between patients with rapid and slow recoveries uncovered significant differences during infection and 4 weeks later for both senses, with rapidly recovered patients having better ratings in both assessments (all $p<$ 0.05), as shown in Figure 1.

Olfactory and gustatory intensity total study group ratings indicated similar fluctuations relative to the VAS scores, with significant decreases during infection and significant improvements 4 weeks after the diagnosis (Table 1).

A comparison of olfactory intensity ratings between subgroups with rapid and slow recoveries showed significantly lower values in the slow-recovery group during infection for bimodal odors; however, this was not the case for unimodal odors (Fig. 2). A similar comparison of subgroups with different recovery patterns for taste intensity ratings showed no significant difference during infection between groups, although the fast-recovery group had slightly better results (Fig. 3a). Four weeks later, the rapid-recovery subgroup had significantly better taste intensity ratings. Taste intensity ratings were not homogeneously reduced, with salty and sweet tastes being more affected, as seen in Figure 3b.

Both VAS and intensity ratings were not correlated with the age of the patients $(r=0.26$, $p=0.76 ; r=0.31, p=0.45$ ). Only 4 patients reported diagnoses of other medical conditions. Thus, no correlation analysis was possible between the presence of comorbidities and the development of olfactory or gustatory dysfunctions. The most common related symptoms of COVID-19 infection were found to be cough (69.5\%), fatigue (45\%), and fever (32\%), with no differences in symptom patterns between subgroups.

Nasal obstruction was reported by 5 out of 30 patients (16.6\%) with chemosensory loss and distributed mainly in the rapid-recovery group ( 4 patients). Similarly, only 3 patients had rhinorrhea (10\%), with 2 of them having a rapid recovery. Olfactory or gustatory dysfunction was not correlated with nasal symptoms such as rhinorrhea or nasal obstruction (all $r<0.21$ ). However, a trend towards significance was found during infection between the olfactory VAS ratings of patients with a rapid olfactory recovery and their nasal obstruction ratings ( $r=$ $0.34, p=0.078$ ). Parosmia and phantosmia were reported by 4 out of 30 patients, and dysgeusia was reported by 3 . 


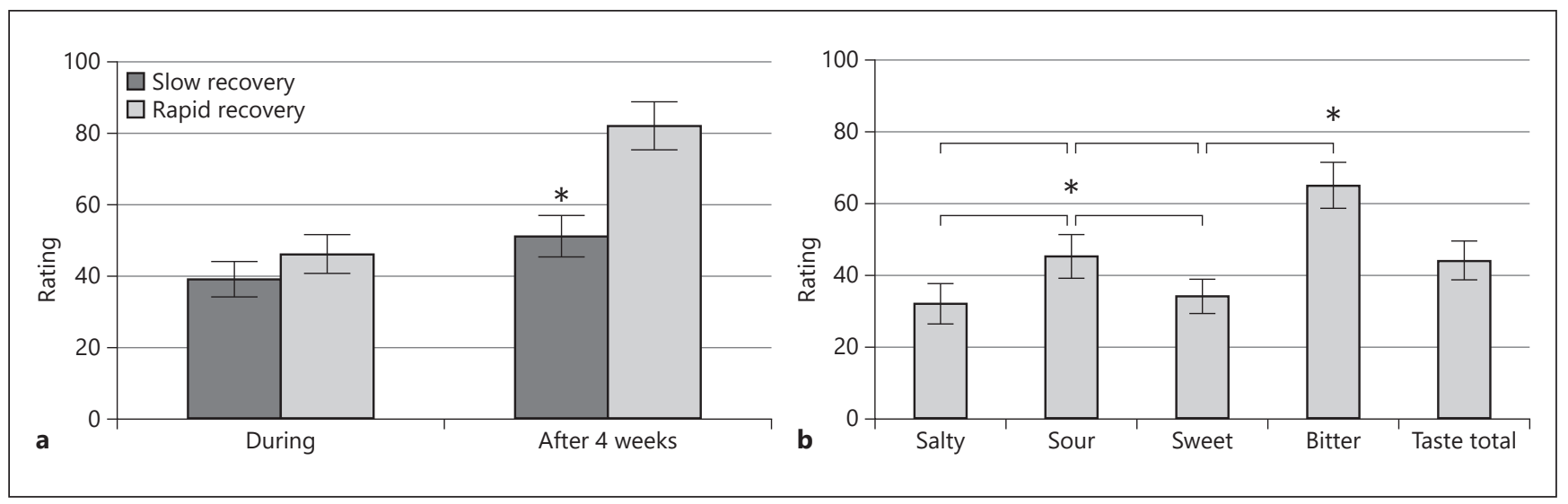

Fig. 3. a Comparison of gustatory function intensity ratings between the rapid- and slow-recovery subgroups. The asterisk indicates statistical significance. b Specific intensity ratings for salty, sweet, sour, bitter, and taste overall intensity scores. The asterisk above the bitter ratings indicates significantly higher values than all other tastes, and the asterisk above the sour ratings indicates significantly higher values than sweet and salty.

\section{Discussion}

Our data indicates that in most cases olfactory and gustatory dysfunction due to COVID-19 infection returns to normal within a few weeks. However, in a smaller but significant percentage of patients ( 1 in 3 patients according to our data), the chemosensory deficit persists. Interestingly, this was not related to the age of the patients, in contrast with the results of Lechien et al. [1]. This could be attributed to the relatively low mean age of our study group, which consisted of only a few patients above the age of 45 years. This fact strengthens the hypothesis that the 2 recovery patterns are perhaps related to different pathophysiological mechanisms and not with the patients' demographic distribution. In addition, the equal distribution of genders in our study is in contrast with other studies presenting a clear female dominance [1,3].

According to our data, the group of patients with a rapid recovery included more patients who reported a loss of smell and taste and nasal obstruction. This fact may partially explain their complete recovery in a few weeks when nasal obstruction and mucosal edema subside, indicating the conductive olfactory loss as the underlying pathology. However, other patients with a total chemosensory loss and no nasal symptoms loss also returned to normal within a few weeks. It is unclear why other patients with the same symptoms did not experience improvement in olfaction and gustation. Currently, some evidence suggests that the COVID-19 virus can enter the nasal epithelium via the receptors ACE2 and TMPRSS2 [12]. These molecules, required for COVID-19 cell entry, are also expressed in the olfactory neuroepithelium. This peripheral involvement suggests that the virus can damage the olfactory mucosa. Perhaps this explains the relatively low intensity ratings of patients reflecting low-threshold performances. Brann et al. [13] suggested that the COVID-19 virus mainly affects nonneural cells of the olfactory epithelium, such as sustentacular cells and horizontal basal cells. The fact that olfactory bipolar cells are not a target of the virus may explain the good prognosis concerning olfactory loss in the majority of cases. The different proportion and cell type of olfactory epithelium damage may be able to result in a different recovery pattern. For example, horizontal basal cells are stem cells that can potentially mature into olfactory neural cells. Disruption of this 
ongoing neurogenesis may result in a more severe olfactory dysfunction compared to that of sustentacular cell damage.

In addition, other studies have suggested that COVID-19 could be neuroinvasive in the central structures of the olfactory system [14]. Mao et al. [15] suggested 3 possible means of entry of SARS-CoV-2 into the brain, i.e.,, via the olfactory nerves in the nasal cavity, through interaction with angiotensin-converting enzyme-2 (ACE-2) in the brain, and via a cytokine storm-induced blood-brain barrier disruption. The above-mentioned variability of the virus's potential entry into and damage to peripheral and central structures of the olfactory system may result in different clinical phenotypes.

We also observed that patients with a slow recovery pattern had lower intensity ratings in odors with strong trigeminal compounds (e.g., mint gum). This may indicate a significant role of the trigeminal nerve damage in clinical phenotypes of the disease. Our results are in accordance with a recently published study by Parma et al. [16], where the authors divided COVID-19 patients into 3 phenotypic clusters, with one of them having a loss of smell, taste, and chemesthesis. Although this phenomenon has been described in previous studies, it was rarely reported by the patients $[17,18]$. The mechanisms are not clear and may be both peripheral and central. The virus could infect surrounding epithelia of trigeminal free endings or their feeding blood vessels $[19,20]$. In addition, there is evidence that intimate central connections between the trigeminal and olfactory systems exist, with overlapping activations in areas such as the piriform cortex, the ventral insula, and the middle frontal gyrus [21]. Considering the potential entry of SARS-CoV-2 into the brain, involvement of the above mentioned areas could explain intranasal trigeminal dysfunction at a central level.

Our results from taste intensity ratings indicate that the reported subjective taste impairment is a result not only of retronasal olfactory loss but also of taste system damage. There is evidence from experimental studies that inflammatory cytokines induced by viral infections can negatively affect the function of taste buds and that viral invasion to taste cells can lead to genetic material exchange between viruses and taste cell subpopulations [22,23]. In addition, similar to olfaction, taste can be also affected by the virus's invasion of the brain, especially involving areas of the central taste system [13,24].

In general, the percentage of patients with mild to moderate disease who report a loss of smell and taste in Greece is not regarded as high compared to those in studies from other countries $[1,25,26]$. The first study from a small cohort of 22 hospitalized patients in this country reported a higher incidence of olfactory loss (59.1\%) after excluding individuals with nasal congestion [27]. Another interesting point from the study demographics was the high percentage of males, in contrast with other studies from Western Europe which reported high percentages of females. This may indicate that geographical differences exist not only because of different health care systems and policies but also due to different infection characteristics. However, the small sample size of our study does not allow us to draw general conclusions regarding the Greek population, and demographic differences may be partially attributed to bias in the recruitment process.

COVID-19 is a new disease and its clinical presentation is most likely associated with different clinical phenotypes [16], which may result in different prognoses.

Undoubtedly, our study has several limitations in terms of its sample size and methodology of assessment. The lack of objective olfactory and gustatory function measurements weakens our results; however, the use of intensity ratings from a home smell-and-taste test bridges the gap with the self-evaluations, which have a low reliability compared to other sensory systems, at least at a population level $[9,10,28]$. 
Konstantinidis et al.: Short-Term Follow-Up of Chemosensory Dysfunction in COVID-19 Patients

\section{Conclusion}

The majority of patients with mild-to-moderate disease and a loss of smell and taste report recovery of their chemosensory deficit in the short term. However, 1 in 3 patients does not, and this appears to not be age related. Based on our results, patients with rapid and slow recoveries present some different characteristics; however, further studies are needed to explore the different phenotypes of the disease and to clarify the underlying mechanisms.

\section{Statement of Ethics}

Investigations were performed according to the Guidelines for Biomedical Studies Involving Human Subjects (Helsinki Declaration). The protocol was approved by the Ethics Committee of Attikon Hospital, Athens, Greece, (513-200320) and by the Ethics Committee of Papageorgiou Hospital, Thessaloniki, Greece, (AP235/18-03-20), and all of the subjects provided written informed consent.

\section{Conflict of Interest Statement}

The authors have no conflict of interests to declare.

\section{Funding Sources}

There is no funding arrangement related to this research.

\section{Author Contributions}

I.K.: study conception; data acquisition, analysis, and interpretation; and drafting, revision, and final approval of this work. A.D. and P.M.: data acquisition and interpretation, and revision and final approval of this work. E.T.: data acquisition and drafting, and revision and final approval of this work. S.S. and S.T.: revision and final approval of this work.

\section{References}

1 Lechien JR, Chiesa-Estomba CM, De Siati DR, Horoi M, Le Bon SD, Rodriguez A, et al. Olfactory and gustatory dysfunctions as a clinical presentation of mild-to-moderate forms of the coronavirus disease (COVID-19): a multicenter European study. Eur Arch Otorhinolaryngol. 2020 Aug;277(8):2251-61.

2 Beltrán-Corbellini Á, Chico-García JL, Martínez-Poles J, Rodríguez-Jorge F, Natera-Villalba E, Gómez-Corral J, et al. Acute-onset smell and taste disorders in the context of COVID-19: a pilot multicentre polymerase chain reaction based case-control study. Eur J Neurol. 2020 Apr;22(9):1738-41.

3 Vaira LA, Salzano G, Deiana G, De Riu G. Anosmia and ageusia: common findings in COVID-19 patients. Laryngoscope. 2020 Jul;130(7):1787.

4 Oleszkiewicz A, Schriever VA, Croy I, Hähner A, Hummel T. Updated Sniffin' Sticks normative data based on an extended sample of 9139 subjects. Eur Arch Otorhinolaryngol. 2019 Mar;276(3):719-28.

5 Landis BN, Welge-Luessen A, Brämerson A, Bende M, Mueller CA, Nordin S, et al. “Taste Strips” - a rapid, lateralized, gustatory bedside identification test based on impregnated filter papers. J Neurol. 2009 Feb;256(2): 242-8.

6 Hummel T, Whitcroft KL, Andrews P, Altundag A, Cinghi C, Costanzo RM, et al. Position paper on olfactory dysfunction. Rhinol Suppl. 2017 Mar;54(26):1-30.

7 Stamps JJ, Bartoshuk LM, Heilman KM. A brief olfactory test for Alzheimer's disease. J Neurol Sci. 2013 Oct; 333(1-2):19-24.

8 Vaira LA, Salzano G, Petrocelli M, Deiana G, Salzano FA, De Riu G. Validation of a self-administered olfactory and gustatory test for the remotely evaluation of COVID-19 patients in home quarantine. Head Neck. 2020 Jul; 42(7):1570-6. 
Konstantinidis et al.: Short-Term Follow-Up of Chemosensory Dysfunction in COVID-19 Patients

9 Kern DW, Schumm LP, Wroblewski KE, Pinto JM, Hummel T, McClintock MK. Olfactory thresholds of the U.S. Population of home-dwelling older adults: development and validation of a short, reliable measure. PLoS One. 2015 Mar;10(3):e0118589.

10 Iravani B, Arshamian A, Ravia A, Mishor E, Snitz K, Shushan S, et al. Relationship between odor intensity estimates and COVID-19 prevalence prediction in a Swedish population. Chem Senses. 2020 May;45(6):bjaa034.

11 Pingel J, Ostwald J, Pau HW, Hummel T, Just T. Normative data for a solution-based taste test. Eur Arch Otorhinolaryngol. 2010 Dec;267(12):1911-7.

12 Hoffmann M, Kleine-Weber H, Schroeder S, Krüger N, Herrler T, Erichsen S, et al. SARS-CoV-2 cell entry depends on ACE2 and TMPRSS2 and is blocked by a clinically proven protease inhibitor. Cell. 2020 Apr; 181(2):271-280.e8.

13 Brann DH, Tsukahara T, Weinreb C, Lipovsek M, Van den Berge K, Gong B, et al. Non-neuronal expression of SARS-CoV-2 entry genes in the olfactory system suggests mechanisms underlying COVID-19-associated anosmia. BioRxIv. 2020, DOI: 10.1101/2020.03.25.009084.

14 Li YC, Bai WZ, Hashikawa T. The neuroinvasive potential of SARS-CoV2 may play a role in the respiratory failure of COVID-19 patients. J Med Virol. 2020 Jun;92(6):552-5.

15 Mao L, Jin H, Wang M, Hu Y, Chen S, He Q, et al. Neurologic manifestations of hospitalized patients with coronavirus disease 2019 in Wuhan, China. JAMA Neurol. 2020 Jun;77(6):683-90.

16 Parma V, Ohla K, Veldhuizen MG, Niv MY, Kelly CE, Bakke AJ, et al.; GCCR Group Author. More than smell COVID-19 is associated with severe impairment of smell, taste, and chemesthesis. Chem Senses. 2020 Jun;bjaa041.

17 Ren Y, Yang L, Guo Y, Xutao M, Li K, Wei Y. Intranasal trigeminal chemosensitivity in patients with postviral and post-traumatic olfactory dysfunction. Acta Otolaryngol. 2012 Sep;132(9):974-80.

18 Pellegrino R, Walliczek-Dworschak U, Winter G, Hull D, Hummel T. Investigation of chemosensitivity during and after an acute cold. Int Forum Allergy Rhinol. 2017 Feb;7(2):185-91.

19 Sungnak W, Huang N, Bécavin C, Berg M, Queen R, Litvinukova M, et al.; HCA Lung Biological Network. SARSCoV-2 entry factors are highly expressed in nasal epithelial cells together with innate immune genes. Nat Med. 2020 May;26(5):681-7.

20 Varga Z, Flammer AJ, Steiger P, Haberecker M, Andermatt R, Zinkernagel AS, et al. Endothelial cell infection and endotheliitis in COVID-19. Lancet. 2020 May;395(10234):1417-8.

21 Hummel T, Oehme L, van den Hoff J, Gerber J, Heinke M, Boyle JA, et al. PET-based investigation of cerebral activation following intranasal trigeminal stimulation. Hum Brain Mapp. 2009 Apr;30(4):1100-4.

22 Wang H, Zhou M, Brand J, Huang L. Inflammation and taste disorders: mechanisms in taste buds. Ann N Y Acad Sci. 2009 Jul;1170(1):596-603.

23 Stone LM, Wilcox CL, Kinnamon SC. Virus-mediated transfer of foreign DNA into taste receptor cells. Chem Senses. 2002 Nov;27(9):779-87.

24 Montalvan V, Lee J, Bueso T, De Toledo J, Rivas K. Neurological manifestations of COVID-19 and other coronavirus infections: A systematic review. Clin Neurol Neurosurg. 2020 Jul;194:105921.

25 Sedaghat AR, Gengler I, Speth MM. Olfactory dysfunction: a highly prevalent symptom of COVID-19 with public health significance. Otolaryngol Head Neck Surg. 2020 Jul;163(1):12-5.

26 Moein ST, Hashemian SM, Mansourafshar B, Khorram-Tousi A, Tabarsi P, Doty RL. Smell dysfunction: a biomarker for COVID-19. Int Forum Allergy Rhinol. 2020 Aug;10(8):944-50.

27 Tsivgoulis G, Fragkou PC, Delides A, Karofylakis E, Dimopoulou D, Sfikakis PP, et al. Quantitative evaluation of olfactory dysfunction in hospitalized patients with Coronavirus [2] (COVID-19). J Neurol. 2020 Aug;267(8): 2193-5.

28 Landis BN, Hummel T, Hugentobler M, Giger R, Lacroix JS. Ratings of overall olfactory function. Chem Senses. 2003 Oct;28(8):691-4. 